\title{
Gene transfer of a TIE2 receptor antagonist prevents pulmonary hypertension in rodents
}

Masakuni Kido, MD

Lingling $\mathrm{Du}, \mathrm{MD}^{\mathrm{a}}$

Christopher C. Sullivan, MS

Reena Deutsch, $\mathrm{PhD}^{\mathrm{b}}$

Stuart W. Jamieson, MB, FRCS ${ }^{a}$

Patricia A. Thistlethwaite, MD, $\mathrm{PhD}^{\mathrm{a}}$
From the Divisions of Cardiothoracic Surgery $^{\mathrm{a}}$ and Biostatistics, ${ }^{\mathrm{b}}$ University of California, San Diego, San Diego, Calif.

Supported by National Institutes of Health grant R01-HL70852, the Charles B. Wang Foundation (PAT), and National Institutes of Health grant M01-RR00827 (RD).

Read at the Eighty-fourth Annual Meeting of The American Association for Thoracic Surgery, Toronto, Ontario, Canada, April 25-28, 2004.

Received for publication March 30, 2004; revisions received Sept 1, 2004; accepted for publication Sept 27, 2004.

Address for reprints: Patricia A. Thistlethwaite, $\mathrm{MD}, \mathrm{PhD}$, Division of Cardiothoracic Surgery, University of California, San Diego, San Diego, CA 92103-8892. E-mail: pthistlethwaite@ucsd.edu.

J Thorac Cardiovasc Surg 2005;129:268-76 $0022-5223 / \$ 30.00$

Copyright (c) 2005 by The American Association for Thoracic Surgery

doi:10.1016/j.jtcvs.2004.09.013
Objectives: Overexpression of angiopoietin 1 in the lung has been associated with human pulmonary hypertension. We hypothesized that inhibiting angiopoietin 1 signaling in the lung by administration of a receptor antagonist would block the development of pulmonary hypertensive vasculopathy in rodent models.

Methods: We injected 2 and $4 \times 10^{10}$ genomic particles of adeno-associated virus containing an extracellular fragment of the TIE2 receptor (AAV-sTIE2) into the pulmonary artery of 60 rats by using adeno-associated virus-lacZ and carrier-injected rats as control animals. Pulmonary hypertension was then induced by each of the following methods: (1) monocrotaline (group 1); (2) angiopoietin 1 expression in pulmonary vascular smooth muscle by adeno-associated virus gene transfer (group 2); or (3) oxygen deprivation (group 3). Animals were sacrificed at serial time points. At each time point, pulmonary artery pressures were measured, and pulmonary angiography was performed. Lungs were harvested for pathologic-molecular analysis.

Results: Each rodent pulmonary hypertension model demonstrated a significant increase in pulmonary artery pressures compared with that seen in control animals $(P<$ .01). Administration of AAV-sTIE2 prevented pulmonary hypertension in the monocrotaline and angiopoietin 1 groups (from $44.6 \pm 2.1$ to $18.8 \pm 1.9 \mathrm{~mm} \mathrm{Hg}$ in the monocrotaline group and from $31.2 \pm 3.7$ to $18.2 \pm 1.8 \mathrm{~mm} \mathrm{Hg}$ in the angiopoietin 1 group, $P<.001$ ) but did not affect pulmonary hypertension in the hypoxia group. Pathologic analysis of group 1 and 2 lungs treated with AAV-sTIE2 demonstrated absence of smooth muscle cell proliferation within arterioles. Pulmonary angiography confirmed a lack of small pulmonary vessel occlusion in group 1 and 2 animals treated with AAV-sTIE2.

Conclusions: Molecular blocking of the interaction between angiopoietin 1 and its endothelial receptor, TIE2, in the lung prevents pulmonary hypertension in 2 animal models of the disease. These experiments suggest a new strategy for understanding pulmonary hypertension based on the molecular biology of the pulmonary vascular wall.

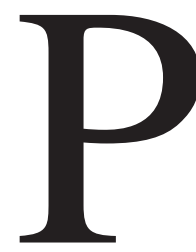
ulmonary hypertension $(\mathrm{PH})$ is a disease characterized by proliferation of vascular smooth muscle cells and asymmetric neointimal endothelial hyperplasia around small pulmonary arteries and arterioles. ${ }^{1}$ Muscle cell proliferation around pulmonary vessels occurs in all forms of $\mathrm{PH}$, including idiopathic disease, as well as that induced by drugs, thromboembolism, or intracardiac shunt. ${ }^{2-4}$ Several lines of evidence have suggested that angiopoietin 1 (Ang-1), a secreted peptide ligand, regulates physiologic vascular smooth muscle proliferation in utero and contributes to pathologic smooth muscle cell proliferation around vessels in PH. First, mice 


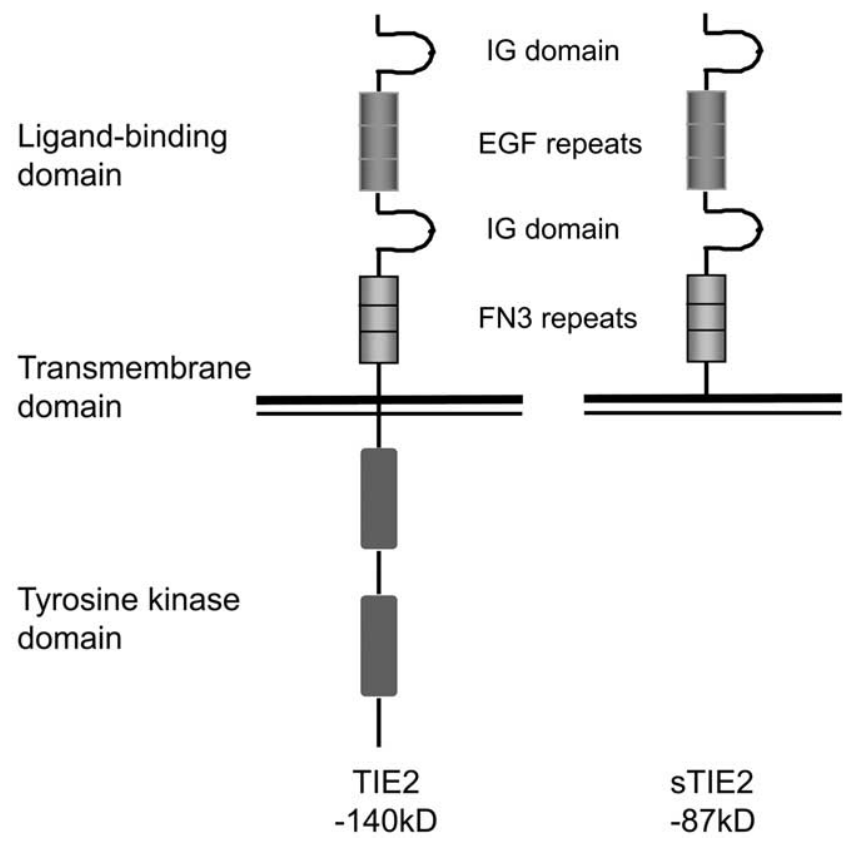

Figure 1. TIE2 receptor with a unique extracellular ligand-binding domain comprised of immunoglobulin (IG) domains, epithelial growth factor (EGF) repeats, and fibronectin-like 3 (FN3) repeats is a transmembrane protein with intracellular tyrosine kinase domain. Soluble TIE2 (sTIE2), a competitive inhibitor of TIE2 activation, is composed of the extracellular ligand-binding domain only.

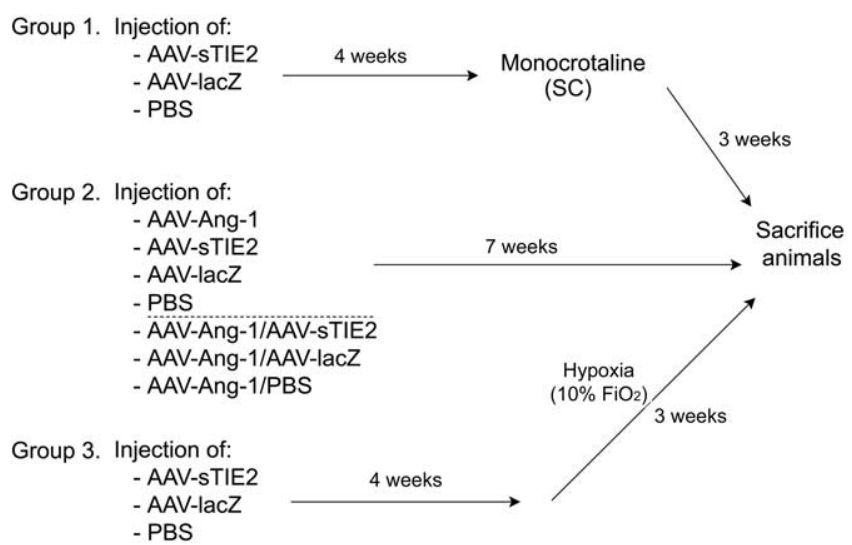

Figure 2. Flow sheet of treatment strategies. AAV, Adeno-associated virus; sTIE2, soluble TIE2; FiO $_{2}$ fraction of inspired oxygen; $S C$, subcutaneous.

lacking Ang-1 die in utero from vascular anomalies resulting from a lack of muscular investment around blood vessels, particularly in the lung. ${ }^{5}$ Second, targeted overexpression of Ang-1 in the lungs of rodents causes clinical and pathologic $\mathrm{PH}$ characterized by severe muscularization of pulmonary arterioles. ${ }^{6,7}$ Third, Ang-1 is constitutively ex-

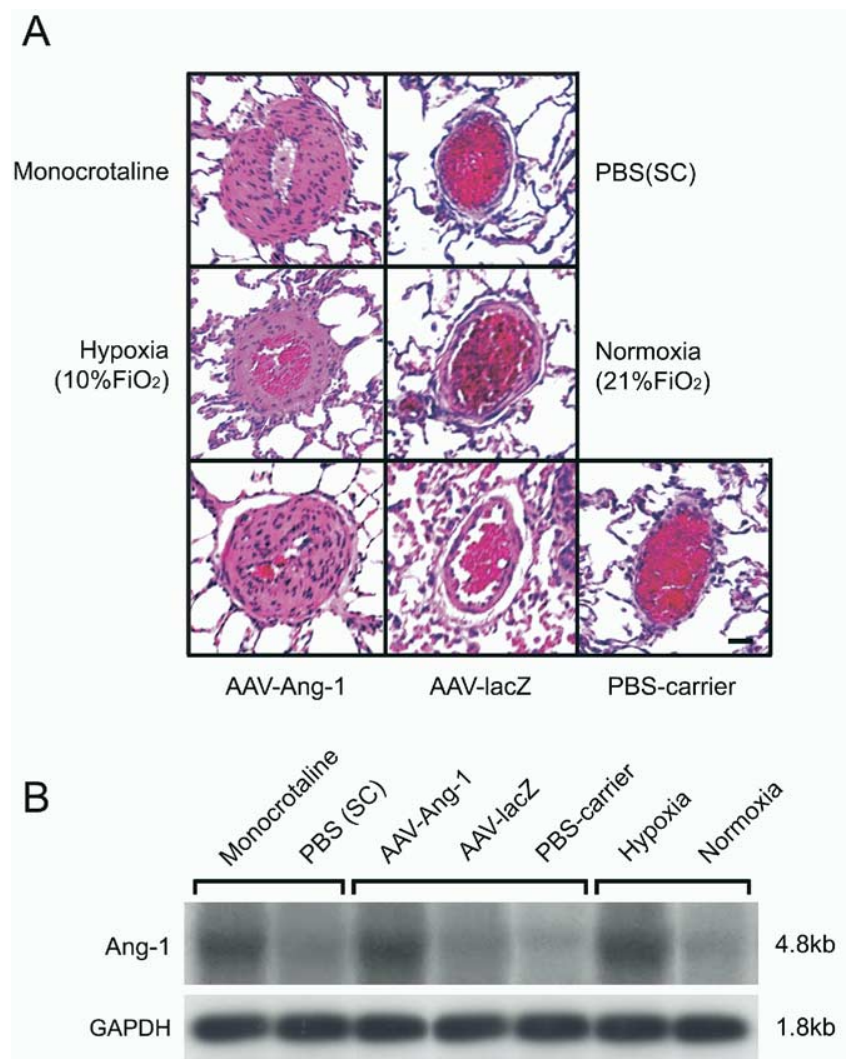

Figure 3. A, Photomicrographs of hematoxylin and eosin-stained rat pulmonary arterioles from 3 animal models of $\mathrm{PH}$ and respective control animals. The top panel compares monocrotalineinduced pathology to control injection of PBS. The middle panel compares hypoxia-induced pathology with normoxic lung. The bottom panel demonstrates AAV-Ang-1-induced pathology compared with that seen in lungs injected with AAV-lacZ or PBScarrier. The scale bar represents $40 \mu \mathrm{m}$. B, Northern blot demonstrating constitutive Ang-1 expression in the lungs of animals with 3 types of PH. Transcript lengths are shown on the right. SC, Subcutaneous injection; $\mathrm{FiO}_{2}$, fraction of inspired oxygen.

pressed in the lungs of patients with different causes of $\mathrm{PH}$ and is absent in normal adult lung tissue. ${ }^{8}$ Taken together, these results suggest a causal role for Ang-1 in the vasculopathy of $\mathrm{PH}$.

Ang-1 exerts its effect through the endothelial-specific tyrosine kinase receptor TIE2. ${ }^{9}$ In lung vascular development, both Ang-1 and TIE2 are expressed in growing blood vessels: Ang-1 is made and secreted by vascular smooth muscle cells and precursor pericytes, whereas TIE2 is a transmembrane receptor expressed on the surface of endothelial cells. ${ }^{10}$ Once development is complete, Ang-1 expression is almost undetectable, whereas TIE2 expression remains constitutive. It is unknown what signals reactivate constitutive Ang-1 gene expression in the pulmonary hypertensive lung. 
TABLE 1. Pressure transduction in 3 models of pulmonary hypertension

\begin{tabular}{|c|c|c|c|c|}
\hline & \multicolumn{2}{|c|}{ PA pressure $(\mathrm{mm} \mathrm{Hg})$} & \multicolumn{2}{|c|}{ Aortic pressure (mm Hg) } \\
\hline & Systolic & Diastolic & Systolic & Diastolic \\
\hline \multicolumn{5}{|l|}{ Group 1} \\
\hline Monocrotaline (SC) & $45.8 \pm 3.7^{*}$ & $22.8 \pm 3.4^{*}$ & $102.4 \pm 5.7$ & $76.4 \pm 2.7$ \\
\hline PBS (SC) & $16.2 \pm 1.5$ & $7.8 \pm 1.3$ & $109.2 \pm 3.4$ & $75.8 \pm 5.1$ \\
\hline \multicolumn{5}{|l|}{ Group 2} \\
\hline \multicolumn{5}{|l|}{ AAV-Ang-1 } \\
\hline $2 \times 10^{10} \mathrm{GP}$ & $33.6 \pm 2.4 \dagger$ & $17.2 \pm 2.6 \dagger$ & $106.8 \pm 10.5$ & $75.2 \pm 5.5$ \\
\hline $4 \times 10^{10} \mathrm{GP}$ & $31.8 \pm 2.6 \dagger$ & $16.6 \pm 2.3 \dagger$ & $106.4 \pm 4.0$ & $74.8 \pm 4.7$ \\
\hline \multicolumn{5}{|l|}{ AAV-sTIE2 } \\
\hline $2 \times 10^{10} \mathrm{GP}$ & $15.8 \pm 1.3$ & $8.0 \pm 1.6$ & $104.8 \pm 4.6$ & $75.2 \pm 3.8$ \\
\hline $4 \times 10^{10} \mathrm{GP}$ & $15.8 \pm 1.6$ & $7.6 \pm 1.1$ & $107.2 \pm 4.9$ & $72.2 \pm 4.0$ \\
\hline \multicolumn{5}{|l|}{ AAV-lacZ } \\
\hline $2 \times 10^{10} \mathrm{GP}$ & $16.0 \pm 1.6$ & $7.0 \pm 1.2$ & $107.6 \pm 3.4$ & $76.8 \pm 4.2$ \\
\hline $4 \times 10^{10} \mathrm{GP}$ & $16.2 \pm 2.4$ & $8.2 \pm 1.9$ & $108.4 \pm 3.9$ & $74.4 \pm 4.8$ \\
\hline PBS-carrier & $16.4 \pm 1.1$ & $7.8 \pm 0.8$ & $101.2 \pm 3.1$ & $73.6 \pm 4.1$ \\
\hline \multicolumn{5}{|l|}{ Group 3} \\
\hline Нурохіа & $42.8 \pm 3.2^{*}$ & $21.4 \pm 3.3^{*}$ & $102.2 \pm 4.3$ & $71.8 \pm 5.8$ \\
\hline Normoxia & $15.2 \pm 1.5$ & $6.0 \pm 1.0$ & $106.8 \pm 7.0$ & $74.4 \pm 2.7$ \\
\hline
\end{tabular}

Values are reported as means \pm SD. $P A$ pressure, Pulmonary arterial pressure; $S C$, subcutaneous; $P B S$, phosphate-buffered saline; $G P$, genomic particles injected per rat; $A A V$, adeno-associated virus; Ang-1, angiopoietin 1; sTIE2, soluble TIE2.

$* P<.01$ compared with control group: PBS (SC) group.

$\dagger P<.001$ compared with 3 control groups: AAV-sTIE2, AAV-lacZ, and PBS-carrier groups.

We hypothesized that disruption of the Ang-1-TIE2 signaling pathway in the lung vasculature would prevent the development of PH. To this end, we examined whether an extracellular fragment of the TIE2 receptor (sTIE2, a peptide retaining the binding site for Ang-1) would serve as a competitive inhibitor of this receptor-ligand interaction and prevent development of $\mathrm{PH}$ when expressed in the pulmonary vascular wall. The effect of sTIE2 gene administration using an adeno-associated viral vector (AAV) to the lung was tested in 3 rodent models of PH.

\section{Materials and Methods}

\section{Vector Construction and Gene Transfer}

A cDNA containing nucleotides 1 to 2236 of the murine TIE2 receptor was generated by standard cloning techniques and named soluble TIE2-cDNA (sTIE2-cDNA). The sTIE2-cDNA codes for the extracellular domain of TIE2 (amino acids 1-744) and encompasses 3 fibronectin type III domains adjacent to 3 epidermal growth factor-like repeats between 2 immunoglobulin-like loops (Figure 1). ${ }^{11}$ The sTIE2 peptide has been shown to block Ang-1 binding to TIE2 in vitro. ${ }^{12}$ The final cDNA was verified by means of sequencing with an ABI-PRISM 3100 Genetic Analyzer (Applied Biosystems, Foster City, Calif) and inserted into the pAAVshuttle plasmid to create pAAV-sTIE2. Large-scale recombinant serotype 2 AAV containing sTIE2, murine Ang-1, or lacZ was produced, purified, and titered as previously reported. ${ }^{13}$ The concentration of the vectors was determined by means of real-time polymerase chain reaction (PCR) with the SYBR Green detection kit (Applied Biosystems). All viral preparations had at least $1 \times$ $10^{11}$ genomic particles $(\mathrm{GP}) / \mathrm{mL}$. Vectors were retested for trans- gene expression by infecting 293T cells. Twelve-week-old Fischer rats (Harlan, San Diego, Calif) were anesthetized with intraperitoneal injection of ketamine $(50 \mathrm{mg} / \mathrm{kg})$-xylazine $(10 \mathrm{mg} / \mathrm{kg})$. Animals were intubated and ventilated with a Harvard rodent ventilator Model 683 (Harvard Apparatus, Holliston, Mass). The heart was exposed through a left thoracotomy at the fourth intercostal space. Injections were made into the main pulmonary artery while occluding the pulmonary veins for 5 seconds. All viral injections consisted of $200 \mu \mathrm{L}$ of phosphate-buffered saline (PBS) $-1 \mathrm{mmol} / \mathrm{L} \mathrm{MgCl}_{2}$ containing the number of viral GP indicated below. Animal experiments were approved by the University of California, San Diego Animal Care Committee and were in compliance with the "Guide for Care and Use of Laboratory Animals" (www.nap.edu/catalog/5140.html, revised 1996).

\section{Animal Models}

Separate treatment groups were used to examine the effects of sTIE2 in 3 models of PH (Figure 2). Group 1 animals received monocrotaline, a pyrrolizidine alkaloid (Sigma Aldrich, St. Louis, Mo), that is known to induce PH 2 to 3 weeks after subcutaneous injection. ${ }^{14}$ Group 2 animals had pulmonary arterial injections of AAV-Ang-1, which is known to induce PH 4 weeks after gene transduction. ${ }^{6}$ Group 3 animals were placed in a hypoxia chamber and allowed to breathe $10 \%$ oxygen for 3 weeks to induce $\mathrm{PH}{ }^{15}$ Experiments for each group were performed in duplicate, with 5 animals per treatment within each subgroup. Groups 1 and 3 received injections of AAV-sTIE2 $\left(2 \times 10^{10}\right.$ and $4 \times 10^{10} \mathrm{GP}$ per rat), AAV-lacZ $\left(2 \times 10^{10}\right.$ and $4 \times 10^{10} \mathrm{GP}$ per rat), or PBS-1 $\mathrm{mmol} / \mathrm{L} \mathrm{MgCl}_{2}$ (PBS-carrier) at week 0 . At week 4 , group 1 animals received subcutaneous injections of either monocrotaline at $60 \mathrm{mg} / \mathrm{kg}$ or diluent alone, whereas group 3 animals were 
A
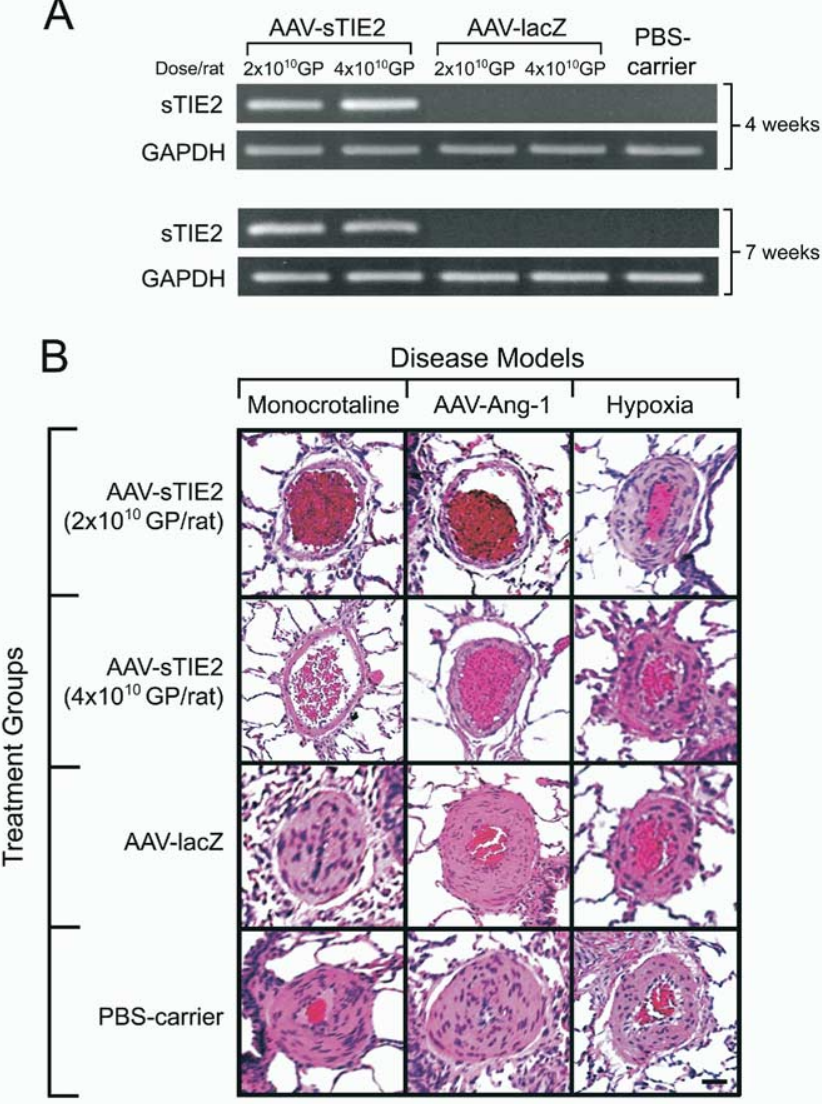

Figure 4. A, Lung specimens 4 and 7 weeks after injection with AAV-sTIE2, AAV-lacZ, or PBS carrier tested for sTIE2 expression by means of RT-PCR. The transgene sTIE2 was constitutively expressed in the lungs of animals injected with AAV-sTIE2 only. $B$, Pathologic changes in lung specimens (hematoxylin and eosin stain). Injection of AAV-sTIE2 prevented pulmonary arteriolar smooth muscle hyperplasia in monocrotaline- and Ang-1-induced models of PH but had no effect on hypoxia-induced PH. The scale bar represents $50 \mu \mathrm{m}$.

exposed to either 3 weeks of hypoxia (10\% fraction of inspired oxygen) in a 14-cubic-feet hypobaric chamber or ambient air. Group 2 received control injections of either AAV-Ang-1 $(2 \times$ $10^{10}$ and $4 \times 10^{10}$ GP per rat), AAV-sTIE $2\left(2 \times 10^{10}\right.$ and $4 \times$ $10^{10} \mathrm{GP}$ per rat), AAV-lacZ $\left(2 \times 10^{10}\right.$ and $4 \times 10^{10} \mathrm{GP}$ per rat $)$, or PBS-carrier, as well as combination injections of AAV-Ang-1 $\left(2 \times 10^{10}\right.$ GP per rat) plus AAV-sTIE2 (in doses of 2 or $4 \times 10^{10}$ GP per rat), AAV-Ang- $1\left(2 \times 10^{10} \mathrm{GP}\right.$ per rat $)$ plus AAV-lacZ (in doses of 2 or $4 \times 10^{10}$ GP per rat $)$, or AAV-Ang- $1\left(2 \times 10^{10} \mathrm{GP}\right.$ per rat) plus PBS-carrier made at week 0 . No treatment was made at week 4. All animals were sacrificed at week 7 .

\section{Hemodynamic Measurements and Pulmonary}

Angiography

Prior to sacrificing animals, pulmonary artery pressures were transduced with a 22-gauge angiocatheter directed into the main pulmonary artery after insertion into the right ventricular outflow

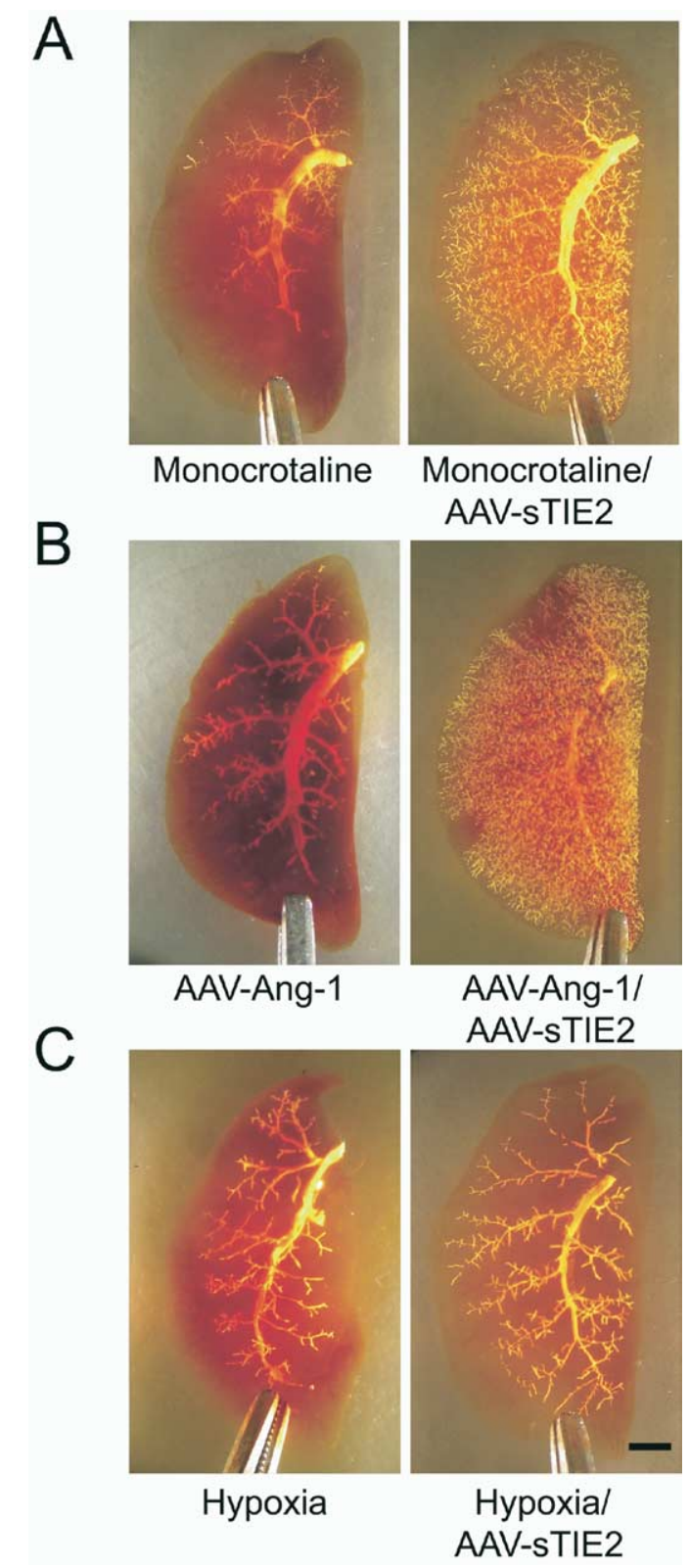

Figure 5. Pulmonary angiograms using the Microfil cast technique of rodent lungs with $\mathrm{PH}$ induced by either monocrotaline (A), constitutive Ang-1 expression (B), or hypoxia (C) treated or untreated with AAV-sTIE2. Note the absence of peripheral vessel staining in the lungs of all 3 models of $\mathrm{PH}$, which is indicative of small-vessel occlusion and normal pulmonary vascular tree in lungs of animals treated with sTIE2. The scale bar represents 25 $\mathrm{mm}$.

tract, while systemic pressures were measured by means of direct aortic transduction (pressure transducer from SpaceLabs, Inc, Issaquah, Wash). For angiography, animals were sacrificed by exsanguination through the abdominal aorta. The pulmonary artery was flushed with $4 \mathrm{~mL}$ of PBS. Lungs were perfused through the pulmonary artery with $0.25 \mathrm{~mL} / \mathrm{min}$ Microfil (Flow Tech, Carver, 
TABLE 2. Morphometric analysis of vascular lesions and pressure measurement

\begin{tabular}{|c|c|c|c|c|c|c|c|c|}
\hline \multirow[b]{2}{*}{ Treatment } & \multirow{2}{*}{$\begin{array}{l}\text { Disease } \\
\text { induction }\end{array}$} & \multirow{2}{*}{$\begin{array}{l}\text { Vessels } \\
\text { occluded }^{*} \\
(\%)\end{array}$} & \multirow{2}{*}{$\begin{array}{c}\text { Vessels with } \\
\text { media } \\
\text { hyperplasia* (\%) }\end{array}$} & \multirow{2}{*}{$\begin{array}{l}\text { Mean no. of } \\
\text { myocytes } \\
\text { vessel wallt }\end{array}$} & \multicolumn{2}{|c|}{ PA pressure (mm Hg) } & \multicolumn{2}{|c|}{ Aortic pressure (mm Hg) } \\
\hline & & & & & Systolic & Diastolic & Systolic & Diastolic \\
\hline \multicolumn{9}{|l|}{ Group 1} \\
\hline $\begin{array}{l}\text { PBS } \\
\text { AAV-sTIE2 }\end{array}$ & lonocrotaline & $36.6 \pm 2.6$ & $82.2 \pm 5.8$ & $34.3 \pm 7.5$ & $44.6 \pm 2.1$ & $24.8 \pm 4.1$ & $105.4 \pm 3.7$ & $72.6 \pm 4.8$ \\
\hline $2 \times 10^{10} \mathrm{GP}$ & Monocrotaline & $3.2 \pm 2.8 \ddagger$ & $10.6 \pm 2.9 \ddagger$ & $12.0 \pm 4.7 \ddagger$ & $18.8 \pm 1.9 \ddagger$ & $9.2 \pm 1.5 \ddagger$ & $106.2 \pm 6.6$ & $76.6 \pm 8.0$ \\
\hline $4 \times 10^{10} \mathrm{GP}$ & Monocr & 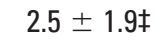 & & & $2.2 \ddagger$ & 8.2 & 07.2 & $74.2 \pm 3.7$ \\
\hline \multicolumn{9}{|l|}{ AAV-lacZ } \\
\hline $2 \times 10^{10} \mathrm{GP}$ & lonocrotaline & $34.2 \pm 6.6$ & $84.5 \pm 4.1$ & $33.6 \pm 9.3$ & $46.8 \pm 3.4$ & $26.2 \pm 3.1$ & $104.6 \pm 4.5$ & $74.2 \pm 5.2$ \\
\hline $4 \times 10^{10} \mathrm{GP}$ & Monocrotaline & $.9 \pm 4.6$ & 77 & $8+90$ & $45.4 \pm 2.1$ & $23.6 \pm 2.9$ & $105.8 \pm 5.3$ & $72.8 \pm 7.6$ \\
\hline \multicolumn{9}{|l|}{ Group 2} \\
\hline $\begin{array}{l}\text { PBS } \\
\text { AAV-sTIE2 }\end{array}$ & AV-Ang-1 & $25.5 \pm 6$ & $.5 \pm$ & $30.4 \pm 6.4$ & $31.2 \pm 3.7$ & $15.2 \pm 2.7$ & $108.6 \pm 3.7$ & $75.4 \pm 4.7$ \\
\hline $2 \times 10^{10} \mathrm{GP}$ & AAV-Ang-1 & $2.9 \pm 1.9 \S$ & $8.3 \pm 2$ & $12.2 \pm 2.9 \S$ & $18.2 \pm 1.8 \S$ & $7.8 \pm 0.8 \S$ & $103.4 \pm 10.3$ & $73.4 \pm 14.0$ \\
\hline $4 \times 10^{10} \mathrm{GP}$ & AAV-Ang-1 & $2.2 \pm 1$ & + & $\S$ & $15.6 \pm 1.5 \S$ & $7.6 \pm 1.1 \S$ & $107.8 \pm 4.3$ & $77.8 \pm 4.7$ \\
\hline \multicolumn{9}{|l|}{ AAV-lacZ } \\
\hline $2 \times 10^{10} \mathrm{GP}$ & AV-Ang-1 & $25.0 \pm 5.2$ & $74.9 \pm 4.6$ & $4+$ & $34.6 \pm 2.3$ & 15.6 & $108.0=$ & $72.2 \pm 3.2$ \\
\hline $4 \times 10^{10} \mathrm{GP}$ & AV-Ang-1 & $25.3 \pm 9.6$ & \pm & \pm & $31.8 \pm 2.9$ & $16.8 \pm 1.6$ & $105.6 \pm 4.6$ & $70.4 \pm 4.5$ \\
\hline \multicolumn{9}{|l|}{ Group 3} \\
\hline $\begin{array}{l}\text { PBS } \\
\text { AAV-s }\end{array}$ & ypoxia & \pm 2.5 & 4.3 & $20.8 \pm 6.8$ & 40 & $20.8 \pm 2.9$ & $96.4 \pm 2.7$ & $70.2 \pm 2.8$ \\
\hline $2 \times 10^{10} \mathrm{GP}$ & Hypoxia & $5.0 \pm 2.6$ & 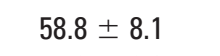 & & $00.7-$ & 19.2 & 97.6 & $70.8 \pm 9.6$ \\
\hline $4 \times 10^{10} \mathrm{GP}$ & Hypoxia & $4.8 \pm 1.9$ & $55.6 \pm 6.6$ & +4.8 & $39.8 \pm 6.5$ & $19.2 \pm 4.2$ & $97.0 \pm 5.1$ & $72.4 \pm 5.3$ \\
\hline \multicolumn{9}{|l|}{ AAV-lacZ } \\
\hline $2 \times 10^{10} \mathrm{GP}$ & ypoxia & $6.3 \pm 2.0$ & $74.8 \pm 5.0$ & $19.6 \pm 5.9$ & $43.6 \pm 4.0$ & $21.8 \pm 1.8$ & $100.4 \pm 2.8$ & $71.8 \pm 3.1$ \\
\hline $4 \times 10^{10} \mathrm{GP}$ & Hypoxia & $6.5 \pm 3.4$ & $75.6 \pm 4.9$ & $19.8 \pm 4.7$ & $42.6 \pm 3.1$ & $22.0 \pm 3.4$ & $98.8 \pm 3.6$ & $70.4 \pm 3.4$ \\
\hline
\end{tabular}

Values are reported as means \pm SD. PA pressure, Pulmonary arterial pressure; PBS, phosphate-buffered saline; AAV, adeno-associated virus; sTIE2, soluble TIE2; $G P$, genomic particles injected per rat.

*Vessels 50-500 $\mu \mathrm{m}$.

$\dagger$ Vessels $<125 \mu \mathrm{m}$.

$\ddagger P<.001$ compared with PBS and AAV-lacZ injection in group 1 .

$\S P<.001$ compared with PBS and AAV-lacZ injection in group 2.

Mass) for 4 minutes with a syringe infusion pump (Harvard Apparatus). Lungs were incubated at $4^{\circ} \mathrm{C}$ for 12 hours, bathed in serial concentrations of ethanol, placed in methyl salicylate, and photographed with a digital camera after magnification (Leica S6D dissecting microscope, Heerbrugg, Switzerland).

\section{Tissue Processing and Histology}

Lungs were fixed in $4 \%$ paraformaldehyde, embedded in paraffin, and sectioned at $5 \mu \mathrm{m}$ thickness. Sections were stained with hematoxylin and eosin and examined by means of digital photomicroscopy at various magnifications to determine the severity of $\mathrm{PH}$. A pathologist blinded to the study reviewed 10 sections per lung and determined, for vessels 50 to $500 \mu \mathrm{m}$ in diameter, the percentage with medial hyperplasia and the percentage occluded, as well as the number of myocytes per vessel wall for vessels less than $125 \mu \mathrm{m}$ in diameter.

\section{RNA Methods}

Reverse transcriptase PCR (RT-PCR) and Northern blotting were performed as previously described. ${ }^{8}$ For RT-PCR, primer pairs used were as follows: viral-specific sTIE2, 5'-CTGCTTGGAC-
CCTTAGTGACATT-3' (derived from a murine sequence) and 5'-GTATCGATAAGCTTGATATCTCTTC-3' (derived from polyadenylation sequence in our viral construct); glyceraldehyde3-phosphate dehydrogenase (GAPDH), ${ }^{9} 5^{\prime}$-CATCATCTCTGCCCCCTCTG-3' and 5'-CCTGCTTCACCACCTTCTTG-3'. For Northern blots, membranes were incubated with phosphorous 32labeled probes generated by RT-PCR from total rat RNA with the following primer pairs: Ang-1, 5'-TGGAGGGAAAACACAAGGAAGAGC-3' and 5'-TAAGGGCGCATTTGCACATACAGT$3^{\prime}$ (derived from mouse/rat sequence); GAPDH, same as above. Primer pairs listed were derived from the National Institutes of Health rat-mouse gene bank at www.NCBI.NLM.NIH.gov by using DNAstar primer select software (DNASTAR, Inc, Madison, Wis). sTIE2 and Ang-1 reaction products were sequenced (ABIPRISM 3100 Genetic Analyzer) to verify amplification of the expected sequences.

\section{Statistical Analysis}

Means \pm SD for direct measurements and means \pm SEM for treatment differences are reported. Wilcoxon Mann-Whitney exact tests performed with StatXact with Cytel Studio software (Version 
6.0 2003; Cytel Software Corp, Cambridge, Mass) were used to test hypotheses of differences. Unadjusted observed significance levels ( $P$ values) are reported.

\section{Results}

\section{Animal Models of PH and Ang-1 Expression}

Pulmonary hypertensive pathology was induced by one of 3 different methods: monocrotaline administration (group 1), injection of AAV-Ang-1 into the main pulmonary artery (group 2), or hypoxic conditioning (group 3). To confirm that pulmonary hypertensive pathology developed in each animal model, rats in each group (and their respective control animals) were sacrificed, and their lungs were examined for pulmonary hypertensive changes (Figure 3,A). All 3 methods induced pulmonary hypertensive vascular changes and measurable $\mathrm{PH}$ (Table 1). Lungs from animals in all groups demonstrated severe muscular hyperplasia in the medial layer of the pulmonary arteries and arterioles measuring less than $500 \mu \mathrm{m}$ in diameter. Northern analysis of tissue after monocrotaline injection, AAV-Ang-1 injection, or oxygen deprivation confirmed high steady-state levels of Ang-1 transcripts in pulmonary hypertensive lungs compared with that seen in control lungs (Figure 3, B). All 3 models of rodent PH were characterized by constitutive expression of Ang-1 mRNA in the lung.

\section{Effect of AAV-sTIE2 on Rodent Pulmonary Hypertensive Pathology}

An experimental flow sheet is described in Figure 2. To determine whether constitutive expression of sTIE2 in the lung could block the development of PH, we injected AAVsTIE2 in 2 doses $\left(2 \times 10^{10}\right.$ GP per rat, 30 total animals; 4 $\times 10^{10} \mathrm{GP}$ per rat, 30 total animals $)$, AAV-lacZ $\left(2 \times 10^{10}\right.$ GP per rat, 30 total animals; $4 \times 10^{10}$ GP per rat, 30 total animals), or an equal volume of PBS-carrier (200 $\mu \mathrm{L}$ per rat, 30 total animals) into the main pulmonary arteries of the rats. RT-PCR analysis at 4 and 7 weeks after injection confirmed that vector-specific sTIE2 transcripts were present in lungs injected with AAV-sTIE2 but were not detected in lungs injected with AAV-lacZ or PBS-carrier (Figure 4, A). After induction of $\mathrm{PH}$ by monocrotaline injection, oxygenation deprivation, or injection of AAVAng-1, animals who had received pretreatment with AAVsTIE2, AAV-lacZ, or PBS-carrier were sacrificed, and their lungs were examined for vascular pathology.

Delivery of AAV-sTIE2 to the lung protected against the development of PH in monocrotaline-injected animals (Figure $4, B)$. Small-vessel medial hyperplasia was present in less than $11 \%$ of blood vessels examined. In contrast, delivery of control virus, AAV-lacZ, had no effect on monocrotaline-induced $\mathrm{PH}$, with greater than $80 \%$ of blood vessels examined displaying medial hyperplasia and 34\% of pulmonary arterioles occluded from smooth muscle cell proliferation (Table 2). The protective effect of sTIE2 occurred with both doses of viral vector administered and occurred irrespective of AAV-sTIE2 viral preparation. In a similar way, AAV-sTIE2 protected rodent lungs against the development of Ang-1-induced PH, blocking the development of small-arteriole muscular hyperplasia (Figure 4, B). Ang- 1 animals constitutively expressing sTIE 2 in the lung had less than $9 \%$ of pulmonary arterioles $(<500 \mu \mathrm{m})$ with medial thickening, whereas Ang-1 animals constitutively expressing lacZ in the lung had greater than $74 \%$ of pulmonary arterioles affected (Table 2).

In contrast, administration of AAV-sTIE2 did not affect the development of hypoxic $\mathrm{PH}$. Severe pulmonary hypertensive lung pathology was seen in animals injected with either AAV-sTIE2 or the control AAV-lacZ vector, and lung pathology in these 2 groups was indistinguishable. Oxygen-deprived animals had medial hyperplasia in approximately $75 \%$ of pulmonary arterioles measuring less than $500 \mu \mathrm{m}$ in diameter, and this process was not attenuated by sTIE2.

\section{STIE2 Prevents PH in 2 Models of the Disease}

For groups 1 and 2 (monocrotaline- and Ang-1-induced $\mathrm{PH}$ ), animals expressing sTIE2 demonstrated a significant decrease in pulmonary systolic and diastolic pressures (Table 2) compared with that seen in control animals, which was reproducible over a 10-minute period before the animals were sacrificed (mean [SEM] decrease, 28.1 [1.1] and 16.2 [1.1] $\mathrm{mm} \mathrm{Hg}$ for pulmonary artery systolic and diastolic pressures, respectively, for group 1 animals treated with AAV-sTIE2; 16.3 [1.1] and 8.5 [0.7] $\mathrm{mm} \mathrm{Hg}$ for pulmonary artery systolic and diastolic pressures, respectively, for group 2 animals treated with AAV-sTIE2). This represented a selective decrease in pulmonary artery pressures because systemic arterial blood pressures were unchanged compared with baseline measurements taken before gene transfer. Pulmonary artery pressures for animals constitutively expressing sTIE2 in the monocrotaline and Ang-1 groups were significantly decreased (all $P<.001$ ) compared with pressures measured in control animals. For group 3 (hypoxic PH), sTIE2 had little effect on pulmonary artery pressures (mean [SEM] change of 3.5 [1.8] and 2.9 [1.3] $\mathrm{mm} \mathrm{Hg}$ for pulmonary artery systolic and diastolic pressures, respectively; $P=.07$ ). Animals in this group manifest significant increases in pulmonary arterial systolic and diastolic pressures compared with systemic pressure.

An in vivo polymer perfusion technique ${ }^{16}$ was used to perform pulmonary arteriography. Group 1 and 2 animals (monocrotaline- and Ang-1-induced $\mathrm{PH}$ ) had pulmonary angiograms demonstrating severe small-vessel pruning similar to that seen in human PH (Figure 5, $A$ and $B$ ). However, animals in these groups treated with AAV-sTIE2 had normal pulmonary angiograms, suggesting that high levels of sTIE2 in the lung blocked the development of disease in these 2 animal models. In contrast, sTIE2 had little protec- 
tive effect on the development of hypoxia-induced $\mathrm{PH}$ and small-vessel occlusion-stenosis. Animals in group 3 (hypoxic $\mathrm{PH}$ ) had pulmonary angiograms displaying diffuse small-vessel stenosis-occlusion, regardless of whether they had been treated with AAV-sTIE2 (Figure 5,C).

\section{Discussion}

$\mathrm{PH}$ is a debilitating and lethal disease that is characterized by small-vessel vasculopathy in the lung. The pathologic hallmark of this disease is polyclonal smooth muscle cell proliferation in the medial layer of small pulmonary arteries and arterioles. The clinical manifestation of this vasculopathy is markedly increased pulmonary artery pressure compared with systemic arterial pressure. Although clinical triggers for this disease are known (hypoxia, increased pulmonary circulation, thromboembolism, and chemical damage), the molecular mechanism responsible for the common lung pathology seen with each of these inciting factors is unknown. Current treatment modalities, such as prostacyclin analogs, endothelin receptor antagonists, and nitric oxide, have been shown to be effective in relieving the pulmonary arteriolar vasoreactive component of this disease; however, none of these drugs is known to alleviate the progressive muscularization of small pulmonary vessels that ultimately leads to right heart failure and death. ${ }^{17,18}$

Our goal has been to block specific gene products that control the development of vasculopathy in $\mathrm{PH}$ to effectively treat or prevent this disease. As a result of the work reported here, we have 4 major conclusions. First, 3 animal models for $\mathrm{PH}$ are characterized by overexpression of the same gene, Ang-1. Second, although they look similar at the pathologic level, animal models for PH do not use the same biochemical-molecular pathway to generate small-vessel muscular hyperplasia. Third, constitutive levels of sTIE2, an antagonist of Ang-1, block the development of PH in 2 animal models of this disease. Our studies suggest that monocrotaline-induced PH works through an Ang-1-related pathway. Fourth, sTIE2 is ineffective in preventing PH induced by oxygen deprivation. The phenotypes observed with gene transfer were not due to viral vector, vector preparation, surgical delivery, or carrier solution used. The AAV constructs used in these experiments did not stimulate perivascular lymphocytic infiltration or inflammatory reaction in lung tissue, as has been seen with gene delivery systems to the lung. ${ }^{19}$ Our results suggest that a molecular strategy that blocks the Ang-1-TIE2 signaling pathway is an effective treatment for preventing rodent $\mathrm{PH}$. From a mechanistic point of view, we believe that blocking Ang1-induced activation of the TIE2 receptor is fundamental to preventing paracrine cellular signaling that culminates in a vascular smooth muscle cell proliferative response.

Recently, our group has shown a link between overexpression of Ang-1 in the lung and the development of $\mathrm{PH}^{6,7}$
Indeed, constitutive Ang-1 expression in the adult rodent lung leads to an exaggerated and organ-specific vascular smooth muscle proliferative response. Most forms of human PH are also characterized by high levels of Ang-1 in vascular endothelium. ${ }^{8}$ This gene expression pattern is not seen in normotensive human lung tissue. We have found that rodent monocrotaline-induced PH is caused by an Ang-1related pathway and can be effectively prevented by high levels of Ang-1-specific antagonist in pulmonary endothelium. In a similar way PH induced by constitutive Ang-1 expression (from viral gene delivery) can be effectively prevented by administration of AAV-sTIE2. These findings link monocrotaline and Ang-1-induced PH in the rodent as having a similar biochemical backbone and response to treatment.

We found that hypoxic $\mathrm{PH}$ is a different disease at the molecular level from other forms of $\mathrm{PH}$ in that it showed little response to treatment with sTIE2. Although Ang-1 levels were increased in lung tissue from animals with this form of disease, we speculate that hypoxic PH does not work solely through an Ang-1-TIE2 pathway. Rather, hypoxic PH may be caused by other parallel molecular pathways that circumvent the obligate need for endothelial TIE2 activation. The reversible nature of hypoxic $\mathrm{PH}$ also contrasts with most human forms of the disease, where smooth muscle cell hyperplasia in pulmonary arterioles is permanent.

Clues as to the pathogenesis of $\mathrm{PH}$ and treatment strategies for this disease come from a growing body of literature about molecular cross-talk that occurs between vascular endothelium and smooth muscle cells. This cell-to-cell communication is regulated by paracrine signals between muscle-secreted peptides and transmembrane tyrosine kinase receptors on endothelial cells. ${ }^{20}$ It is widely believed that endothelial cells, through secretion of factors, modulation of cell-surface proteins, or both, regulate the proliferation of smooth muscle cells in their vicinity. Ang-1, the muscle-secreted ligand most studied to date, is known to bind the endothelial-restricted receptor TIE2, resulting in tyrosine phosphorylation and activation of this receptor into an active tyrosine kinase. ${ }^{21}$ Ang-1 signaling through TIE2 is known to result in the following: (1) the release of the paracrine smooth muscle cell growth factor 5-hydroxytryptamine (serotonin) by vascular endothelial cells ${ }^{7}$; (2) the transcriptional downregulation of the BMPR1a gene, ${ }^{8}$ whose sister receptor, BMPR2, is characterized by haploid mutation in inherited forms of human $\mathrm{PH}^{22}$; and (3) the upregulation of the apoptosis inhibitor survivin through the serine threonine kinase pathway Akt, thereby protecting endothelium from programmed cell death. ${ }^{23,24}$ Each of these downstream intracellular pathways may contribute to the pathogenesis of this disease. Efforts to block TIE2 intracellular endothelial signaling in $\mathrm{PH}$ at other more 
downstream events might also prove to be effective treatment strategies for this disease.

Several limitations to this study deserve comment. First, animal treatments were based on the transduction of a specific gene product (a truncated extracellular fragment of the TIE2 receptor sTIE2) for only 50 days after gene delivery in a vector system (AAV) that does not achieve high levels of transgene expression until approximately 1 month after gene delivery. ${ }^{12}$ This is an inherent limitation of gene transfer experiments and does not necessarily reflect the natural time course of the human disease. Second, no animal model completely mimics the irreversible human forms of this disease. Monocrotaline administration not only results in PH but also in panlung inflammation and sporadic development of adenocarcinomas. ${ }^{25}$ Ang-1-induced PH causes smooth muscle cell vascular hyperplasia but rarely results in the development of plexiform lesions in the lung, which are commonly seen in end-stage human PH. ${ }^{7,26}$ Finally, animals with hypoxia-induced $\mathrm{PH}$ have reversal of clinical disease when they are returned to normoxic conditions. Despite these limitations, we show that delivery of a vector containing sTIE2 to the lung vasculature was successful at preventing the development of $\mathrm{PH}$ in 2 models of this disease in rodents. Our results suggest that molecular targeting of the Ang-1-TIE2 gene axis in pulmonary endothelium might be a novel strategy for the treatment of PH based on the genetic profile of the pulmonary vascular wall in this disease. The full potential of therapeutic modulation of the Ang-1-TIE2 pathway will require a greater understanding of the biology of this signaling system in both the normal and pathologic adult lung vasculature.

We thank Paul L. Wolf, MD, of the San Diego VA Medical Center for help with analysis of lung slides.

\section{References}

1. Pietra GG, Edwards WD, Kay JM, Rich S, Kernis J, Schloo B, et al. Histopathology of primary pulmonary hypertension. A qualitative and quantitative study of pulmonary blood vessels from 58 patients in the National Heart, Lung, and Blood Institute, Primary Pulmonary Hypertension Registry. Circulation. 1989;80:1198-206.

2. Jamieson SW, Kapelanski DP. Pulmonary endarterectomy. Curr Probl Surg. 2000;37:165-252.

3. Rich S, Rubin L, Walker AM, Schneeweiss S, Abenhaim L. Anorexigens and pulmonary hypertension in the United States: results from the surveillance of North American pulmonary hypertension. Chest. 2000; 117:870-4.

4. Granton JT, Rabinovitch M. Pulmonary arterial hypertension in congenital heart disease. Cardiol Clin. 2002;20:441-57.

5. Suri C, Jones PF, Patan S, Bartunkova S, Maisonpierre PC, Davis S, et al. Requisite role of angiopoietin-1, a ligand for the TIE2 receptor, during embryonic angiogenesis. Cell. 1996;87:1171-80.

6. Chu D, Sullivan CC, Du L, Cho AJ, Kido M, Wolf PL, et al. A new animal model for pulmonary hypertension based on the overexpression of a single gene, angiopoietin-1. Ann Thorac Surg. 2004;77:449-57.

7. Sullivan CC, Du L, Chu D, Cho AJ, Kido M, Wolf PL, et al. Induction of pulmonary hypertension by an angiopoietin 1/TIE/serotonin pathway. Proc Natl Acad Sci U S A. 2003;100:12331-6.
8. Du L, Sullivan CC, Chu D, Cho AJ, Kido M, Wolf PL, et al. Signaling molecules in nonfamilial pulmonary hypertension. $N$ Engl $J$ Med. 2003;348:500-9.

9. Davis S, Aldrich TH, Jones PF, Acheson A, Compton DL, Jain V, et al. Isolation of angiopoietin-1, a ligand for the TIE2 receptor, by secretion-trap expression cloning. Cell. 1996;87:1161-9.

10. Thistlethwaite PA, Lee SH, Du LL, Wolf PL, Sullivan C, Pradhan S, et al. Human angiopoietin gene expression is a marker for severity of pulmonary hypertension in patients undergoing pulmonary thromboendarterectomy. J Thorac Cardiovasc Surg. 2001;122:65-73.

11. Peters KG, Kontos CD, Lin PC, Wong AL, Rao P, Huang L, et al. Functional significance of tie2 signaling in the adult vasculature. Recent Prog Horm Res. 2004;59:51-71.

12. Lin P, Buxton JA, Acheson A, Radziejewski C, Maisonpierre PC, Yancopoulos GD, et al. Antiangiogenic gene therapy targeting the endothelium-specific receptor tyrosine kinase Tie2. Proc Natl Acad Sci U S A. 1998;95:8829-34.

13. Chu D, Sullivan CC, Weitzman MD, Du L, Wolf PL, Jamieson SW, et al. Direct comparison of efficiency and stability of gene transfer into the mammalian heart using adeno-associated virus versus adenovirus vectors. J Thorac Cardiovasc Surg. 2003;126:671-9.

14. Cowan KN, Heilbut A, Humpl T, Lam C, Ito S, Rabinovitch M. Complete reversal of fatal pulmonary hypertension in rats by a serine elastase inhibitor. Nat Med. 2000;6:698-702.

15. Murata T, Sato K, Hori M, Ozaki H, Karaki H. Decreased endothelial nitric-oxide synthase (eNOS) activity resulting from abnormal interaction between eNOS and its regulatory proteins in hypoxia-induced pulmonary hypertension. J Biol Chem. 2002;277:44085-92.

16. Coral-Vazquez R, Cohn RD, Moore SA, Hill JA, Weiss RM, Davisson RL, et al. Disruption of the sarcoglycan-sarcospan complex in vascular smooth muscle: a novel mechanism for cardiomyopathy and muscular dystrophy. Cell. 1999;98:465-74.

17. Olschewski H, Simonneau G, Galie N, Higenbottam T, Naeije R, Rubin LJ, et al. Inhaled iloprost for severe pulmonary hypertension. N Engl J Med. 2002;347:322-9.

18. Sitbon O, Badesch DB, Channick RN, Frost A, Robbins IM, Simonneau G, et al. Effects of the dual endothelin receptor antagonist bosentan in patients with pulmonary arterial hypertension: a 1-year follow-up study. Chest. 2003;124:247-54.

19. Gautam A, Waldrep CJ, Densmore CL. Delivery systems for pulmonary gene therapy. Am J Respir Med. 2002;1:35-46.

20. Thurston G. Role of angiopoietins and Tie receptor tyrosine kinases in angiogenesis and lymphangiogenesis. Cell Tissue Res. 2003;314:61-8.

21. Jones N, Chen SH, Sturk C, Master Z, Tran J, Kerbel RS, et al. A unique autophosphorylation site on Tie2/Tek mediates Dok-R phosphotyrosine binding domain binding and function. Mol Cell Biol. 2003;23:2658-68

22. Deng Z, Morse JH, Slager SL, Cuervo N, Moore KJ, Venetos G, et al. Familial primary pulmonary hypertension (gene PPH1) is caused by mutations in the bone morphogenetic protein receptor-II gene. Am J Hum Genet. 2000;67:737-44.

23. Kim I, Kim HG, So JN, Kim JH, Kwak HJ, Koh GY. Angiopoietin-1 regulates endothelial cell survival through the phosphatidylinositol 3'-kinase/Akt signal transduction pathway. Circ Res. 2000;86:24-9.

24. Papapetropoulos A, Fulton D, Mahboubi K, Kalb RG, O'Connor DS, $\mathrm{Li} \mathrm{F}$, et al. Angiopoietin-1 inhibits endothelial cell apoptosis via the Akt/survivin pathway. J Biol Chem. 2000;275:9102-5.

25. Ghodsi F, Will JA. Changes in pulmonary structure and function induced by monocrotaline intoxication. Am J Physiol. 1981;240:H149-55.

26. Cool CD, Rai PR, Yeager ME, Hernandez-Saavedra D, Serls AE, Bull TM, et al. Expression of human herpesvirus 8 in primary pulmonary hypertension. $N$ Engl J Med. 2003;349:1113-22.

\section{Discussion}

Dr Ross M. Ungerleider (Portland, Ore). I am curious whether you could tell us what you think it is about hypoxia-induced $\mathrm{PH}$ that makes it so much different than the other forms?

Dr Thistlethwaite. Hypoxic PH is a very interesting phenomenon in that it is a disease that is completely reversible, unlike 
almost every other type of $\mathrm{PH}$, which is permanent. Basically, there are, in the field of $\mathrm{PH}$ research, 2 components to this disease: one is a reactive component of vasoconstriction, and one, a later component, is that of smooth muscle cell proliferation in the vascular wall.

I think that the hypoxic PH model is probably more based on vasoconstriction and less muscular thickening than some of the other models of PH, both human and rodent, and I think, fundamentally, it is a different disease at a genetic level.

Dr Robert C. Robbins (Stanford, Calif). You had to give this before, so I would say it is a great intervention to prevent the PH. What about after PH is already developed in these models? Have you tried giving this intervention to see whether it would regress or improve? Or, for example, in the middle of your models of progression to $\mathrm{PH}$, can it at least arrest the process once it starts, or is it going to be required to be given up front? It really is not going to have much clinical implication if that is the case.

Dr Thistlethwaite. Those are very interesting questions. Our experiments have been limited by the technology of AAV. As most of you know, AAV expresses a transgene about a month after delivery, and therefore in our first experiments or treatment, we wanted to have maximal transgene expression at the time that the disease was clinically induced. We have not done further studies of inducing the disease and then delivering the transgene, but obviously this is something that we will want to look at in the future. 